Published in final edited form as:

Nano Lett. 2016 February 10; 16(2): 856-863. doi:10.1021/acs.nanolett.5b03035.

\title{
Oxidation of Carbon Nanotubes in an Ionizing Environment
}

\author{
Ai Leen Koh ${ }^{1,}$, , Emily Gidcumb ${ }^{2}$, Otto Zhou ${ }^{2,3}$, and Robert Sinclair ${ }^{4,}$ \\ ${ }^{1}$ Stanford Nano Shared Facilities, Stanford University, Stanford, California 94305, USA \\ ${ }^{2}$ Department of Applied Physical Sciences, University of North Carolina at Chapel Hill, Chapel \\ Hill, North Carolina 27599, USA \\ ${ }^{3}$ Department of Physics and Astronomy, University of North Carolina at Chapel Hill, Chapel Hill, \\ North Carolina 27599, USA \\ ${ }^{4}$ Department of Materials Science and Engineering, Stanford University, Stanford, California \\ 94305, USA
}

\begin{abstract}
In this work, we present systematic studies on how an illuminating electron beam which ionizes molecular gas species can influence the mechanism of carbon nanotube oxidation in an environmental transmission electron microscope (ETEM). We found that preferential attack of the nanotube tips is much more prevalent than for oxidation in a molecular gas environment. We establish the cumulative electron doses required to damage carbon nanotubes from $80 \mathrm{keV}$ electron beam irradiation in gas versus in high vacuum. Our results provide guidelines for the electron doses required to study carbon nanotubes within or without a gas environment, to determine or ameliorate the influence of the imaging electron beam. This work has important implications for in situ studies as well as for the oxidation of carbon nanotubes in an ionizing environment such as that occurring during field emission.
\end{abstract}

\section{Graphical abstract}

*Corresponding Author: alkoh@stanford.edu (A.L.K.), bobsinc@stanford.edu (R.S.). Author Contributions

The manuscript was written through contributions of all authors. All authors have given approval to the final version of the manuscript. CONFLICT OF INTEREST

O.Z. has equity ownership and serves on the board of directors of XinRay Systems which develops and manufactures CNT field emission x-ray sources.

Supporting Information. Video clip showing structural changes in a carbon nanotube when illuminated under the electron beam at room temperature with $0.7 \mathrm{mbar}_{2}$. Attack of side wall and tip of nanotube upon exposure to electron beam in nitrogen gas at room temperature. Reaction of an eight-walled CNT in oxygen gas at room temperature. This material is available free of charge via the Internet at http://pubs.acs.org. 


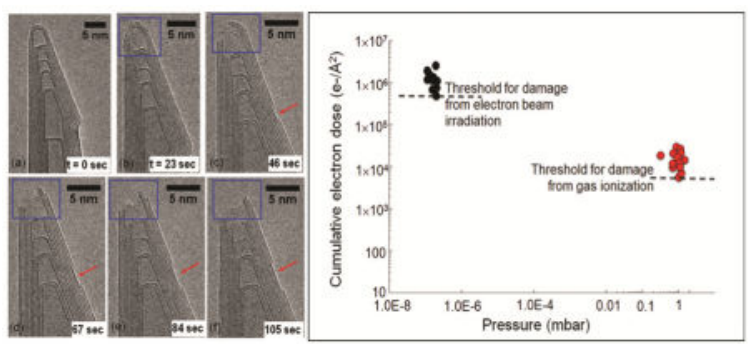

\section{Keywords}

Environmental TEM; aberration-corrected TEM; carbon nanotubes; gas ionization; oxidation

One of the major applications of carbon nanotubes (CNTs) ${ }^{1}$ is for field-emission sources ${ }^{2-4}$ in image displays, ${ }^{5-8}$ high-intensity X-ray tubes ${ }^{9-11}$ for medical applications, ${ }^{12-14}$ and electron sources for electron microscopes. ${ }^{15-17}$ CNTs have large length-to-diameter aspect ratios, which are useful for achieving high local emission fields. Furthermore, they also have excellent structural properties, high electrical and thermal conductivities, and lower fabrication costs and emission threshold fields compared to other emitter materials. ${ }^{18-21}$

Carbon nanotube-based field emission sources are housed in vacuum chambers with a base pressure of $\sim 10^{-7}$ mbar or better. ${ }^{12,22-24}$ It is known that the field emission current significantly reduces under non-ideal vacuum conditions. For example, Bonard and coworkers ${ }^{25}$ found that the emission performance of multiwalled nanotube films was directly related to the chamber pressure. Dean and coworkers ${ }^{23}$ studied the effect of high partial pressures of different gases on the field emission characteristics of nanotubes and showed that nanotube emitter currents decreased rapidly when they were exposed to an oxygen environment. These latter authors have hypothesized that degradation comes about from ion bombardment or etching of the nanotubes as induced through ionization of the surrounding gas by the field-emission current. Other studies of carbon nanotubes in an oxygen environment, not in situ, suggested that the nanotubes are consumed first at the hemispherical cap at their ends, and subsequently by the removal of graphitic planes along the lengths of the tubes. ${ }^{26,27}$

The transmission electron microscope (TEM) is an indispensable tool for characterizing the structure of CNTs owing to its sub-nanometer spatial resolution. In situ TEM, which enables real time observations of material reactions at atomic level, clearly provides direct information not available by other techniques. ${ }^{28}$ There is both a wide range of in situ experiments, from heating ${ }^{29}$ and cooling ${ }^{30}$ to mechanical deformation ${ }^{31}$ and electrical biasing, ${ }^{32}$ but the biggest new contribution is in the control of the specimen environment, be it liquid or gas [ ${ }^{33-37}$, and their references therein]. Environmental TEM (ETEM), which allows gases to be introduced into the otherwise high vacuum of a TEM, is emerging as a powerful technique to study the structural changes in nanomaterials in gaseous environments. ${ }^{37-40}$ In a recent investigation of carbon nanotube oxidation in an ETEM, with the imaging electron beam blanked during $\mathrm{O}_{2}$ exposure, we showed that the molecular oxidation of individual carbon nanotubes under mild oxidation conditions $(1.5 \mathrm{mbar})$ and 
elevated temperatures (up to $520^{\circ} \mathrm{C}$ ) occurred layer by layer, starting with the outermost wall. ${ }^{41}$ In contrast to what had previously been reported, ${ }^{26,27}$ we found that the tube cap was not preferentially prone to oxidation. ${ }^{41,42}$ Recently, Liu and coworkers studied the chiral dependent reactivity of individual carbon nanotubes using ex situ oxidation in air at elevated temperatures and found partial destruction in the nanotube side walls, ${ }^{43}$ a finding similar to our study. Our first experiments were designed to be quasi in situ, performed in the absence of an imaging electron beam and therefore any gaseous ionization it may cause, and structural changes in carbon nanotubes were documented after they have interacted with molecular gas species which had then been purged from the chamber. ${ }^{41}$

In situ observations using a TEM require an illuminating electron beam on the specimen. It is implicit that when carbon nanotubes field emit in less stringent vacuum conditions, any neighboring gas species are likely to be ionized as well. The topic of ionization in gases due to electron impact was first extensively studied from the 1930s to 1960s owing to significant potential applications in plasma physics and vacuum technology. ${ }^{44-48}$ These experiments were typically performed using an electron beam of energy of up to $\sim 1000 \mathrm{eV}^{47,48}$ placed perpendicular to an ionization tube in which gas particles (atoms and molecules) were accelerated. With the discovery of carbon nanotubes and the exciting potential applications that it has brought about, irradiation effects in carbon nanotubes due to electrons and ions ${ }^{49-51}$ are also being widely researched. However, none of the studies to-date has focused on the influence of gas ionization from the electron beam on solid-gas reaction studies in an ETEM. In addition to the beam damage that may be observed in conventional (high-vacuum) TEM, ionization of gas molecules as a result of the interaction between fast electrons and gas also leads to increased reactivity. ${ }^{52}$ To understand the effects of ionized gas molecules near the carbon nanotubes, and to simulate the more highly ionized environment which is expected during field emission conditions we have repeated our observations in the presence of the imaging beam. This also provides the opportunity to record the events at the atomic level in situ as they occur, and to systematically and quantitatively study the influence of the imaging electron beam in gas-solid reactions. It will be seen that the behavior is quite different from that in molecular oxygen.

The CNTs investigated in this work were multiwalled carbon nanotubes (MWNT) synthesized using the arc-discharge method. ${ }^{53}$ Previous studies have shown that they have between four and 34 walls, with outer diameters between 6 and $31 \mathrm{~nm}$, and are highly resistant to mild oxidation at elevated temperatures. ${ }^{41}$ All experiments were carried out at room temperature using a FEI 80-300 kV environmental TEM (FEI Company) equipped with a spherical aberration (Cs) corrector in the image-forming (objective) lens and a monochromator. An accelerating voltage of $80 \mathrm{kV}$, which is thought to be below the threshold energy for knock-on damage for carbon, ${ }^{54}$ was used. The Cs image corrector was adjusted to a Cs value close to $0 \mu \mathrm{m}$, and all images were acquired at slightly underfocus conditions. TEM images were acquired using an Ultrascan 1000 XP CCD camera at binning two setting $(1024 \times 1024$ pixels). During the TEM image acquisition, the corresponding electron dose flux (measured in units of number of electrons per square Angström per second, $\mathrm{e}^{-} / \AA^{2} \cdot \mathrm{sec}$ ) was also recorded. This parameter had been calibrated for the instrument using an analytical TEM holder with a Faraday cup. For gas ionization effects due to the electron beam, oxygen gas and nitrogen gas of research grade 6.0 (99.9999\%) purity 
(Praxair Inc.) were used. The gas pressure was adjusted to between $0.5 \mathrm{mbar}$ and $1.0 \mathrm{mbar}$ for the experiments, and the actual pressure in the microscope chamber was monitored using an Edwards Barocell 600 capacitance manometer with which the microscope is equipped. Continuous recording at 20 frames per second frame speed was achieved using CamStudio software. Frames were extracted and analyzed using VirtualDub. Electron energy loss spectra (EELS) were taken in TEM diffraction mode using a Quantum 966 Gatan Imaging Filter (GIF). The convergence and collection semi-angles were $7.4 \mathrm{mrad}$ and $11.9 \mathrm{mrad}$, respectively. A dispersion of $0.25 \mathrm{eV} /$ pixel and an acquisition time of $0.2 \mathrm{sec}$ per spectrum were used for the EELS collection.

When molecular gas species are exposed to the electron beam during an ETEM experiment, ions of the gaseous species can form by electron displacement due to the collision and or charge transfer from the incident electron beam to the gas molecules. Collision between the oxygen particles (neutral and charged) and carbon atoms can lead to displacement or removal of $\mathrm{C}$ atoms. Figures 1 and 2 show the most commonly observed phenomena in the present study, neither of which occurred without the imaging beam. ${ }^{41}$ The images in Figure 1 were extracted from a video clip (Supplementary Video S1) which was recorded when a multiwalled carbon nanotube was illuminated under the electron beam with $0.7 \mathrm{mbar}_{2}$ in the environmental cell at room temperature, using an electron dose flux of $640 \mathrm{e}^{-} / \AA^{2}$. $\mathrm{sec}$. After the nanotube had been exposed to the beam and gas for 23 seconds, its tip started to degrade, as observed by the region indicated by the blue box in the Figure. After 46 seconds (Figure 1c), the cap was fully open, as a result of $\mathrm{C}$ atoms being oxidized or etched away. One of its side walls was also damaged within the duration of this recording, indicated by the red arrow in Figures 1(e)-(f). Based on the images which show the removal of a hemispherical cap, we estimated the rate of oxygen ionization at room temperature to be 50 carbon atoms/second.

After the tube cap opened, the inner walls became more susceptible to oxidation due to higher curvature, as seen in Fig. 1(f) to (j). Within four minutes (240 sec) of oxygen exposure under an illuminated electron beam, the nanotube had lost its tip and part of its side- and inner-walls. This increased reactivity is clearly the result of gas ionization arising from interaction between the electron beam and oxygen, as carbon and oxygen are known not to react with each other at room temperature. In molecular oxidation studies carried out with the electron beam blanked, there is no evident oxidation of the arc discharge CNTs at room temperature. ${ }^{41}$

An additional outcome in which the ionized gas reacts with the specimen is elucidated in Figure 2, which shows another MWNT that had been exposed to $0.7 \mathrm{mbar}_{2}$. It appeared that the combination of electron beam irradiation and oxygen particles partially removed the outermost graphitic layer of the nanotube by amorphizing it, as indicated by the blue arrow in Figure 2(a). Several seconds later, part of the outermost two layers were destroyed, resulting in the formation of stepped edges in the nanotube Figure 2(b). Panels 2(c) - (g) show that a similar process occurs on the other side of the same nanotube, which eventually led to the removal of the side wall. The rate of removal of carbon atoms from the side wall ionization was calculated to be about 45 atoms/second. The results presented in Figures 1 and 2 are representative of those made additionally on other nanotubes. We repeated the 
experiment using nitrogen gas and under similar electron dose flux. $\mathrm{N}_{2}$ has a similar mass to oxygen, is inert and should not react with carbon. However, we found that the nanotubes were also affected in a similar manner when they were exposed to $\mathrm{N}_{2}$ in the presence of an electron beam (Figure 3), including both side wall amorphization as in Fig. 3 and tube cap opening (Supplementary Figure S1), with $\mathrm{C}$ atoms being etched away at about the same rate as that in oxygen. In contrast, the rate of molecular oxidation based on our previous work ${ }^{41}$ was found to be about 10 carbon atoms/minute (or 0.16 atom/second) at $300{ }^{\circ} \mathrm{C}$. We attribute this behavior to gas ionization from the electron beam as well as momentum transfer from the gas and ion species to the nanotubes. Zhu and coworkers had previously reported that the bombardment of MWNTs using $3 \mathrm{keV}$ Ar ions resulted in the appearance of carbon dangling bonds, and a gradual amorphization of the carbon network during ion irradiation. ${ }^{55}$

In the gas environment of an ETEM, the ionization of the molecular gas species by the electron beam can also give rise to characteristic electron energy loss (EEL) spectra corresponding to the ionization energies of the gas atoms, as shown in our earlier work. ${ }^{41}$ EELS in the ETEM has been used to measure the composition of gases (including $\mathrm{H}_{2}$ ) inside a TEM environmental cell to an accuracy of about $15 \% .{ }^{56}$ More recently, EELS has been employed to detect and quantify catalytic products directly inside the environmental cell of the TEM. ${ }^{57}$ In an oxygen molecule, the electron impact process can lead to the formation of a positive ion which is described by the equation: $e+\mathrm{O}_{2} \rightarrow \mathrm{O}_{2}{ }^{+}+2 e^{45,46}$ Negative ions can also be formed in collisions with molecules in two-body collisions at low pressures by dissociative attachment and ion pair formation. ${ }^{48}$ Due to the high energy and velocity of the incident electron beam in our experiments, we believe it is more likely for the source electron to displace another electron from the gaseous species, leading to a positivelycharged ionized gas.

We also utilized TEM-EELS to gain insight into the damage mechanism of carbon nanotubes by ionized oxygen. This was achieved by condensing the size of the electron beam onto a closed end of a nanotube which is about $30 \mathrm{~nm}$ in diameter, and monitoring the changes in its carbon $\mathrm{K}$ ionization edge over time. In core loss EELS, the ionization energy provides elemental information on the atoms present in the sample, and the spectrum shape can be considered to be a 'fingerprint' of the material. Spectra I to V in Figure 4(a) show the background subtracted C K EEL spectra of a CNT acquired during this process. The time interval between each of these five spectra is estimated to be about 15 seconds. In each of the spectra, the $\mathrm{O} \mathrm{K}$ edge at $\sim 530 \mathrm{eV}$ arising from the oxygen gas is clearly evident. The nanotube was gradually destroyed as it was exposed to oxygen, leading to a decrease in the total carbon K signal. We attempted to quantify the change in the $\pi^{*}$ and $\sigma^{*}$ carbon ratio, by applying a $3 \mathrm{eV}$ integration window over the $\pi^{*}(284.5-287.5 \mathrm{eV})$ and $\sigma^{*}(291.5-294.5 \mathrm{eV})$ peaks, and taking the ratio of the integrated electron counts. We found this value to be fairly consistent during the course of the data acquisition $\left(\frac{N_{\pi *}}{N_{\sigma *}}=0.52 \pm 0.05\right)$, indicating little change in the carbon bonding.

The inset of Fig. 4(a) shows background-subtracted spectra I to IV and an undamaged nanotube in the energy range of 285 to $300 \mathrm{eV}$, where additional broad peaks centered around $289-290 \mathrm{eV}$ (marked with *) are observed in spectra I to IV. Braun and coworkers 
used EELS and near edge X-ray absorption fine structure spectroscopy (NEXAFS) to study soot and had assigned energy losses of carboxyl $(\mathrm{CO}-\mathrm{OH})$, carbonyl $(\mathrm{C}=\mathrm{O})$ and $\mathrm{CO}_{2}$ (or carbonate in general) groups at $288.4 \mathrm{eV}, 289.2 \mathrm{eV}$ and $290.1 \mathrm{eV}$, respectively. ${ }^{57}$ Crozier and workers had previously utilized EELS in the ETEM to quantify catalytic products and had attributed the $289 \mathrm{eV}$ peak to the $\mathrm{C} \pi *$ peak from $\mathrm{CO}_{2} \cdot{ }^{58}$ Since these energies of the carbonyl and carbonate groups are similar to our observations, we hypothesize that they may likely have come from dangling bonds from the broken CNT edges and tips after oxidation, or from $\mathrm{CO}_{2}$ product species. However, since EELS is generally thought to be a lesser energy resolution technique compared to NEXAFS, the exact nature of the group(s) remains to be determined.

A comparison of the C K EEL spectra from an undamaged CNT, a CNT which has been attacked by oxygen and from amorphous carbon (taken from the support film of the TEM grid) is presented in Figure 4(b). We noted that the $\sim 289 \mathrm{eV}$ feature is clearly present only in the $\mathrm{O}_{2}$-reacted CNT. The EEL spectrum of the etched CNT indicates amorphization in the $\mathrm{sp}^{3}$ carbon state in the $295-320 \mathrm{eV}$ range, which is consistent with the high resolution image appearance such as those presented in Figure 2.

Damage in carbon nanotubes can also result from knock-on damage from the incident electron beam. Even though an incident electron beam energy of $80 \mathrm{kV}$, which is thought to be below the $86 \mathrm{kV}$ threshold for knock-on damage, ${ }^{54}$ was utilized in our study, several groups had reported that this threshold energy can be reduced if there are contaminants or defects in the carbon nanotubes. ${ }^{59,60}$ Nanotube diameters as well as their chirality are thought to play a role as well. ${ }^{60,61}$ As a control experiment, we also subjected MWNTs to continuous electron irradiation under high vacuum conditions $\left(\sim 1 \times 10^{-7}\right.$ mbar pressure $)$, and an image was recorded every minute. Figure 5 shows the structural change in a carbon nanotube acquired under high vacuum conditions over time, using an electron dose flux of $694 \mathrm{e}^{-} / \AA^{2} \cdot \mathrm{sec}$ which was similar to the gas ionization experiments presented. After about 18 min of electron irradiation, part of the outermost wall of the nanotube was removed (Figure $5 d)$. Subsequently, its neighboring walls at the tube cap were also removed, as shown in the image in Figure 5(e) which was acquired after 26 min of electron beam exposure. It should be noted that such exposure times are quite long for normal TEM imaging of CNTs which remain undamaged during conventional characterization experiments.

There is a growing trend of in situ environmental studies. ${ }^{28,37}$ Despite the increasing significance in this field, very little attention has been paid to the influence of gas ionization from the electron beam on solid-gas reaction studies in an ETEM. In addition to the beam damage that may be observed in conventional (high-vacuum) TEM, ionization of gas molecules as a result of the interaction between fast electrons and gas leads to increased reactivity. ${ }^{52}$ It is therefore very important to evaluate the results to determine or to ameliorate the influence of the imaging electron beam There are only a few groups (including ourselves) who recognize the importance and influence of the electron beam in gas experiments, and who have proceeded to design and execute gas experiments by taking into consideration the effect of electron dose rate, ${ }^{62-64}$ total electron dose, ${ }^{63}$ and performing beam-blanking studies. ${ }^{41,65}$ In the present work, we quantified the influence of the electron beam on carbon nanotube damage at different pressures in the ETEM column by plotting the 
cumulative electron dose (expressed in units of number of electrons per square Angström) versus pressure, for the nanotubes that were investigated in this study (14 datasets in ETEM, and 11 datasets in high vacuum). This is shown in Figure 6. There is a two order of magnitude difference in the cumulative electron dose to cause onset of visible damage to the carbon nanotubes at room temperature arising from gas pressure of up to $1 \mathrm{mbar}\left(1.3 \times 10^{4}\right.$ $\mathrm{e}^{-} / \AA^{2}$ median cumulative electron dose) compared to continuous electron beam irradiation in high vacuum $\left(1.2 \times 10^{6} \mathrm{e}^{-} / \AA^{2}\right.$ median cumulative electron dose). Based on our findings, we determined the lower limit, threshold cumulative electron dose for damage from electron beam irradiation in high vacuum $\left(5.1 \times 10^{5} \mathrm{e}^{-} / \AA^{2}\right)$ and from gas ionization $\left(6.2 \times 10^{3} \mathrm{e}^{-/}\right.$ $\AA^{2}$ ). A lower cumulative dose at the respective pressures causes no visible deterioration of the CNTs.

This work shows that the behavior of carbon nanotubes in an oxygen environment of about 1 mbar is quite different when an imaging electron beam in an ETEM is illuminating the area of interest compared to when the beam is blanked. Thus the CNT caps are preferentially removed as well as parts of the outer side walls, and local carbon amorphization can take place as well, at room temperature. When no imaging beam is used, with similar gas pressures, temperatures about $300{ }^{\circ} \mathrm{C}$ are required to oxidize the CNTs and the tube caps are not seen to be preferentially etched (Figure 7 , and also [41,42]).

Our interpretation of these observations focuses on the presence of ionized oxygen species (most likely $\mathrm{O}_{2}{ }^{+}$) created by the electron beam, as demonstrated for instance by the oxygen ionization energy loss in EELS data. The CNTs are electrically grounded by their contact with the TEM specimen holder. Thus, the oxygen ions will be attracted to the CNTs by image forces and their average path will follow the effective electric field lines to the nanotubes. This is the reverse situation from that of field emission or field ionization whereby the charged species travel away from a negatively biased sharp emitter for the former or a positively charged tip for the latter (e.g. [66]). It is well-known that in the latter situations that the electrons or ions depart close to radially and that the electric field, being proportional to the applied voltage divided by the local tip radius, is highest at the points of highest curvature of the tip, which has been recently confirmed by electron holography. ${ }^{67}$ Moreover, here the ions would be attracted over a large solid angle at the approximately hemispherical cap, again the reverse of the field ionization process. Field-ion images for instance typically cover an angle of more than $120^{\circ} 66$ showing that the high electric field emanates from an angular range of $\frac{4}{3} \pi$ steradians or more.

In our situation, the highest image field and the point of convergence clearly occur at the nanotube tip, and so damage by the incoming ion current would be expected to be highest there. This is shown in Figure 1 and also in Supplemental Figure S2, an eight-walled nanotube which is the thinnest tube that we studied in these experiments, and which has a relatively small radius of curvature. It can be seen in the latter that the damage clearly occurs most severely at the tip. The challenge in studying thinner tubes is that they tend to vibrate more under the electron beam, and are less stable especially after material has been removed during the initial stages of ionization. There is also an enhanced field along the nanotube shank, owing to its own curvature, and so ion attack is also seen in this location. This is further supported by the observation of CNT damage created in a nitrogen environment 
under the electron beam: the ionized nitrogen species would damage the nanotubes in the same way, while carbon is inert to reaction with molecular nitrogen. On the other hand, for molecular oxygen which is present in the ETEM when the imaging beam is prevented from reaching the specimen chamber, there is no preferential attraction to the nanotube cap. As the area exposed to the gas by the side walls is so much larger than the area of the cap (increasing linearly with the length of the nanotube) it is then more likely that oxidation is seen more commonly there than at the cap itself. It has been suggested that the curved nature of the cap leading to the presence of pentagonal carbon atom rings, as in fullerenes, gives rise to a lower stability to oxidation, ${ }^{27}$ but in our observations, the much larger surface area of the side walls seems to be dominant. It is interesting that fewer wall nanotubes, and inside walls of broken nanotubes, are oxidized more rapidly than larger diameter multiwall nanotubes, ${ }^{41}$ and this likely also arises from the bond distortion associated with the small radius of curvature of those fully hexagonal, $\mathrm{sp}^{2}$-bonded graphene layers.

These results are clearly important to the strategy of studying solid-gas interactions in situ in an ETEM. We have chosen to obtain information on the natural behavior upon heating in a molecular gas environment by blanking the electron beam while the gas species is present in the specimen chamber, and taking sequential images with no gas present. However, the demonstration that there is a threshold level for damage under the electron beam shows that it may be possible to record images under low dose conditions (e.g. using a defocused imaging beam) and with fast acquisition speeds which are possible with the new generation of direct electron detection cameras. ${ }^{68,69}$ This approach may allow investigation of the natural behavior as well but as with all in situ TEM experiments the results need to be checked against those where the electron beam cannot influence the results.

On the other hand, the observations of the behavior in an ionized gas environment have their own importance. For instance, the high electron current created by field emitting CNTs will also ionize any gaseous species locally which will then be attracted to the field emitter tip by the enhanced field associated with the applied negative voltage at a highly curved surface. This might well lead to opening up of the cap tip as shown in Figure 1, which will further influence the field emission process. Indeed, it has been suggested ${ }^{4}$ that such a phenomenon is responsible for increased field emission current for CNTs in a non-optimal vacuum environment, which is consistent with the experimental observations which we have made here. Of course, the direct observation of field emitting CNTs in a gaseous environment is required to determine the exact behavior, but the insight gained in the present work is relevant to understanding the possible reactions involved.

In the gas environment in the ETEM, gas ionization from the imaging electron beam can influence carbon nanotube oxidation by rapidly displacing and amorphizing carbon atoms at both caps and sidewalls at room temperature. The ionization process and damage mechanism are independent of the gas reactivity to carbon, occurring for both oxygen and nitrogen. With a high enough cumulative electron dose, carbon nanotubes can also be damaged by continuous electron beam irradiation in a high vacuum setting. Our findings show that there is a two order of magnitude difference in cumulative electron dose to damage carbon nanotubes in high vacuum and in a gas environment up to $1 \mathrm{mbar}$ pressure. By understanding the influence of the imaging electron beam in a gas environment, and 
determining the threshold level for damage, we anticipate that experimental conditions which delineate its influence can be established which will enable us to study the CNT field emission process in situ in an ETEM.

\title{
MATERIALS AND METHODS
}

CNT Synthesis and TEM Specimen Preparation. The CNTs used in this study were synthesized by an arc discharge method. ${ }^{52}$ Holey carbon molybdenum TEM grids grids (300 mesh, $30 \mathrm{~nm}$ thick, Pacific Grid-Tech) were used for the ETEM experiments. For TEM specimen preparation, the nanotubes were suspended in ethyl alcohol. The vials were bath sonicated for about $10 \mathrm{~min}$ or until agglomerates broke up. Then the nanotube suspension was drop cast onto the TEM grids and wicked dry using filter paper.

\section{Supplementary Material}

Refer to Web version on PubMed Central for supplementary material.

\section{Acknowledgments}

\author{
Funding Sources
}

This work is supported by the following funding sources: National Cancer Institute grants CCNE U54CA-119343 (O.Z.), R01CA134598 (O.Z.), and CCNE-T U54CA151459-02 (R.S.).

The authors thank Dr. Bo Gao of Xintek for providing the raw CNT materials used in this study. Use of the Stanford Nano Shared Facilities is appreciated.

\section{References}

1. Iijima S. Nature. 1991; 354:56-58.

2. Chernozatonskii LA, Gulyaev YV, Kosakovskaya ZY, Sinitsyn NI, Torgashov GV, Zakharchenko Yu F, Fedorov EA, Val'chuk VP. Chem Phys Lett. 1995; 233:63-68.

3. de Heer WA, Châtelain A, Ugarte D. Science. 1995; 270:1179-1180.

4. Rinzler AG, Hafner JH, Nikolaev P, Lou L, Kim SG, Tomimek D, Nordlander P, Colbert DT, Smalley RE. Science. 1995; 269:1550-1553. [PubMed: 17789445]

5. Wang QH, Setlur AA, Lauerhaas JM, Dai JY, Seelig EW, Chang RPH. Appl Phys Lett. 1998; 72:2912-2913.

6. Choi WB, Chung DS, Kang JH, Kim HY, Jin YW, Han IT, Lee YH, Jung JE, Lee NS, Park GS, et al. Appl Phys Lett. 1999; 75:3129-3131.

7. Wang QH, Yan M, Chang RPH. Appl Phys Lett. 2001; 78:1294-1296.

8. Lee NS, Chung DS, Han IT, Kang JH, Choi YS, Kim HY, Park SH, Jin YW, Yi WK, Yun MJ, et al. Diamond and Related Materials. 2001; 10:265-270.

9. Sugie H, Tanemura M, Filip V, Iwata K, Takahashi K, Okuyama F. Appl Phys Lett. 2001; 78:25782580.

10. Yue GZ, Qiu Q, Gao B, Cheng Y, Zhang J, Shimoda H, Chang S, Lu JP, Zhou O. Appl Phys Lett. 2002; 81:355-357.

11. Zhou, O.; Lu, JP. US Patent. US6553096. 2005.

12. Liu Z, Yang G, Lee YZ, Bordelon D, Lu J, Zhou O. Appl Phys Lett. 2006; 89:103111.

13. Cao G, Burk LM, Lee YZ, Calderon-Colon X, Sultana S, Lu J, Zhou O. Med Phys. 2010; 37:53065312. [PubMed: 21089765]

14. Qian X, Tucker A, Gidcumb E, Shan J, Yang G, Calderon-Colon X, Sultana S, Lu J, Zhou O, Spronk D, et al. Med Phys. 2012; 39:2090-2099. [PubMed: 22482630] 
15. Bonard JM, Salvetat JP, Stockli T, Forro L, Chatelain A. Appl Phys A. 1999; 69:245-254.

16. de Jonge N, Lamy Y, Schoots K, Oosterkamp TH. Nature. 2002; 420:393-395. [PubMed: 12459778]

17. de Jonge N. Adv in Imaging and Electron Phys. 2009; 156:203-233.

18. Cheng Y, Zhou OCR. Phys. 2003; 4:1021-1033.

19. Zhou J, Xu NS, Deng SZ, Chen J, She JC, Wang ZL. Adv Mater. 2003; 15:1835-1840.

20. Zhu W, Bower C, Kochanski GP, Jin S. Solid-State Electron. 2001; 54:921-928.

21. Chueh YL, Chou LJ, Cheng SL, He JH, Wu WW, Chen L. J Appl Phys Lett. 2005; 86:133112.

22. Purcell ST, Vincent P, Journet C, Binh VT. Phys Rev Lett. 2002; 88:15502.

23. Dean KA, Chalamala BR. Appl Phys Lett. 1999; 75:3017-3019.

24. Bonard JM, Salvetat JP, Stöckli T, de Heer WA, Forró L, Châtelain A. Appl Phys Lett. 1998; 73:918-920.

25. Bonard JM, Maier F, Stöckli T, Châtelain A, de Heer WA, Salvetat JP, Forró L. Ultramicroscopy. 1998; 73:7-15.

26. Tsang SC, Harris PJF, Green MLH. Nature. 1993; 362:520-522.

27. Ajayan PM, Ebbesen TW, Ichihashi T, Iijima S, Tanigaki K, Hiura H. Nature. 1993; 362:522-525.

28. Sinclair R. MRS Bulletin. 2013; 38:1065-1071.

29. Adhikari H, Marshall AF, Goldthorpe IA, Chidsey CED, McIntyre PC. ACS Nano. 2007; 1:415422. [PubMed: 19206662]

30. De Graef M, Willard MA, McHenry ME, Zhu Y. IEEE Trans Magnetics. 2001; 37:2663-2665.

31. Shan ZW, Mishra RK, Syed Asif SA, Warren OL, Minor AM. Nature Mater. 2008; 7:115-119. [PubMed: 18157134]

32. Meister S, Schoen DT, Topinka MA, Minor AM, Cui Y. Nano Lett. 2008; 8:4562-4567. [PubMed: 19367977]

33. Zheng H, Smith RK, Jun YW, Kisielowski C, Dahmen U, Alivisatos AP. Science. 2009; 324:1309. [PubMed: 19498166]

34. Zheng H, Claridge SA, Minor AM, Alivisatos AP, Dahmen U. Nano Lett. 2009; 9:2460. [PubMed: 19408927]

35. Gai PL, Sharma R, Ross FM. MRS Bulletin. 2008; 33:107-114.

36. de Jonge N, Ross FM. Nature Nano. 2011; 6:695-704.

37. Koh, AL.; Lee, SC.; Sinclair, R. A Brief History of Controlled Atmosphere Transmission Electron Microscopy. In: Hansen, TW.; Wagner, JB., editors. Controlled Atmosphere Transmission Electron Microscopy - Principles and Practice. Springer International Publishing; 2016. p. 3-43.

38. Boyes ED, Gai PL. Ultramicroscopy. 1997; 67:219-232.

39. Hansen TW, Wagner JB, Dunin-Borkowski RE. Mat Sci and Tech. 2010; 26:1338-1344.

40. Sharma RJ. Mater Res. 2005; 20:1695-1707.

41. Koh AL, Gidcumb E, Zhou O, Sinclair R. ACS Nano. 2013; 7:2566-2572. [PubMed: 23360330]

42. Sinclair R, Kempen PJ, Chin RW, Koh AL. Adv Eng Mat. 2014; 16:476-481.

43. Liu B, Jiang H, Krasheninnikov AV, Nasibulin AG, Ren W, Liu C, Kauppinen EI, Cheng HM. Small. 2013; 9:1379-1386. [PubMed: 23495250]

44. Smith PT. Phys Rev. 1930; 36:1293-1302.

45. Fite WL, Brackmann RT. Phys Rev. 1959; 113:815-816.

46. Fite WL, Brackmann RT. Phys Rev. 1958; 112:1141-1151.

47. Rapp D, Englander-Golden P. J Chem Phys. 1965; 43:1464-1479.

48. Rapp D, Briglia DD. J Chem Phys. 1965; 43:1480-1489.

49. Banhart F. Rep Prog Phys. 1999; 62:1181-1221.

50. Krasheninnikov AV, Nordlund K. Nucl Instr and Meth in Phys Res B. 2004; 216:355-366.

51. Krasheninnikov AV, Banhart F. Nature Mater. 2007; 6:723-733. [PubMed: 17906658]

52. Wagner, JB.; Beleggia, M. Gas-Electron Interaction in the ETEM. In: Hansen, TW.; Wagner, JB., editors. Controlled Atmosphere Transmission Electron Microscopy - Principles and Practice. Springer International Publishing; 2016. p. 63-94. 
53. Ebbesen TW, Ajayan PM. Nature. 1992; 358:220-222.

54. Smith BW, Luzzi DE. J Appl Phys. 2001; 90:3509-3515.

55. Zhu Y, Yi T, Zheng B, Cao L. Appl Surf Sci. 1999; 137:83-90.

56. Crozier PA, Chenna S. Ultramicroscopy. 2011; 111:177-185. [PubMed: 21333854]

57. Braun A, Huggins FE, Shah N, Chen Y, Wirick S, Mun SB, Jacobsen C, Huffman GP. Carbon. 2005; 43:117-124.

58. Chenna S, Crozier PA. ACS Catal. 2012; 2:2395-2402.

59. Kotakoski J, Arenal R, Kurasch S, Jiang H, Skakalova V, Stephan O, Krasheninnikov AV, Kauppinen EI, Kaiser U, Meyer JC, et al. ACS Nano. 2012; 6:8837-8846. [PubMed: 23009666]

60. Warner JH, Schäffel S, Zhong G, Rümmeli MH, Büchner B, Robertson J, Briggs GAD. ACS Nano. 2009; 3:1557-1563. [PubMed: 19462964]

61. Krasheninnikov AV, Banhart F, Li JX, Foster AS, Nieminen RM. Phys Rev B. 2005; 72:125428.

62. Simonsen SB, Chorkendorff I, Dahl S; Skoglundh M, Sehested J, Helveg S. J Am Chem Soc. 2010; 132:7968-7975. [PubMed: 20481529]

63. Kuwauchi Y, Yoshida H, Akita T, Haruta M, Takeda S. Angew Chem Int Ed Engl. 2012; 124(31): 7849-7853.

64. Helveg S, Kisielowski CF, Jinschek JR, Specht P, Yuan G, Frei H. Micron. 2014; 68:176-185. [PubMed: 25245867]

65. Hansen TW, Delariva AT, Challa SR, Datye AK. Acc Chem Res. 2013; 46(8):1720-1730. [PubMed: 23634641]

66. Müller, EW.; Tsong, TT. Field Ion Microscopy Principles and Applications. American Elsevier Publishing Company Inc; New York: 1969.

67. Migunov V, London A, Farle M, Dunin-Borkowski RE. J Appl Phys. 2015; 117:134301.

68. Bammes BE, Rochat RH, Jakana J, Chen DH, Chiu W. J Struct Biol. 2012; 177:589-601. [PubMed: 22285189]

69. Li X, Mooney P, Zheng S, Booth CR, Braunfeld MB, Gubbens S, Agard DA, Cheng Y. Nature Meth. 2013; 10:584-590.

Nano Lett. Author manuscript; available in PMC 2017 February 10. 

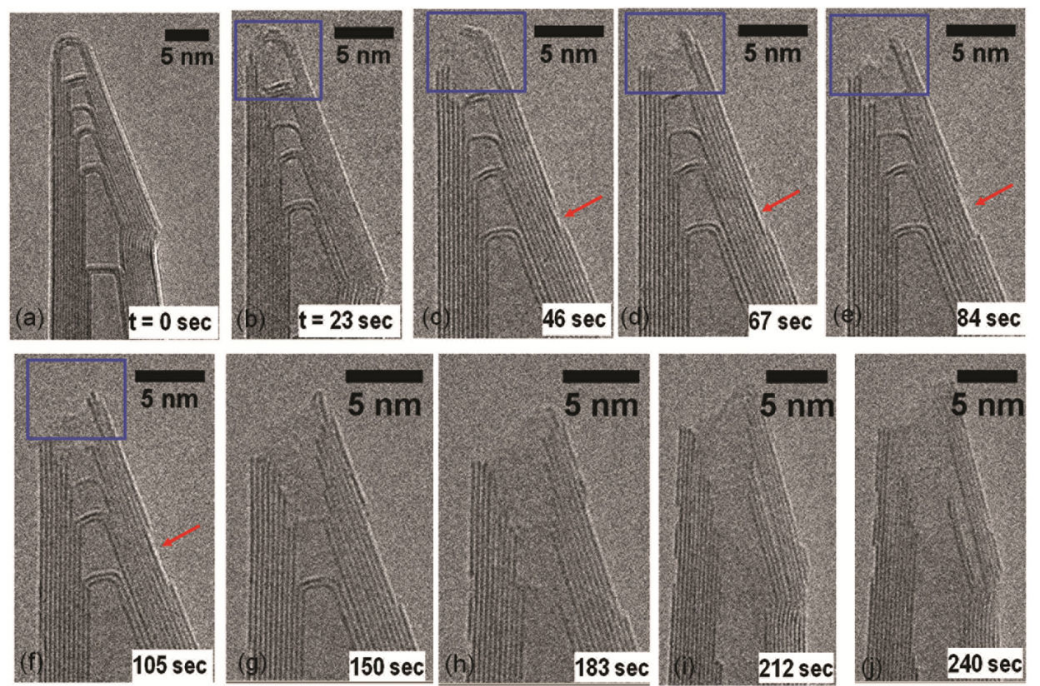

Figure 1.

Time series of TEM images showing damage to the cap and side wall of a nanotube during exposure to the electron beam in $0.7 \mathrm{mbar}_{2}$. The nanotube cap started to degrade after 23 $\sec (\mathrm{b})$, and became fully open after $46 \mathrm{sec}$ (c). The side walls were also damaged (red arrow), as were the inner walls (f to $\mathrm{j}$ ). 

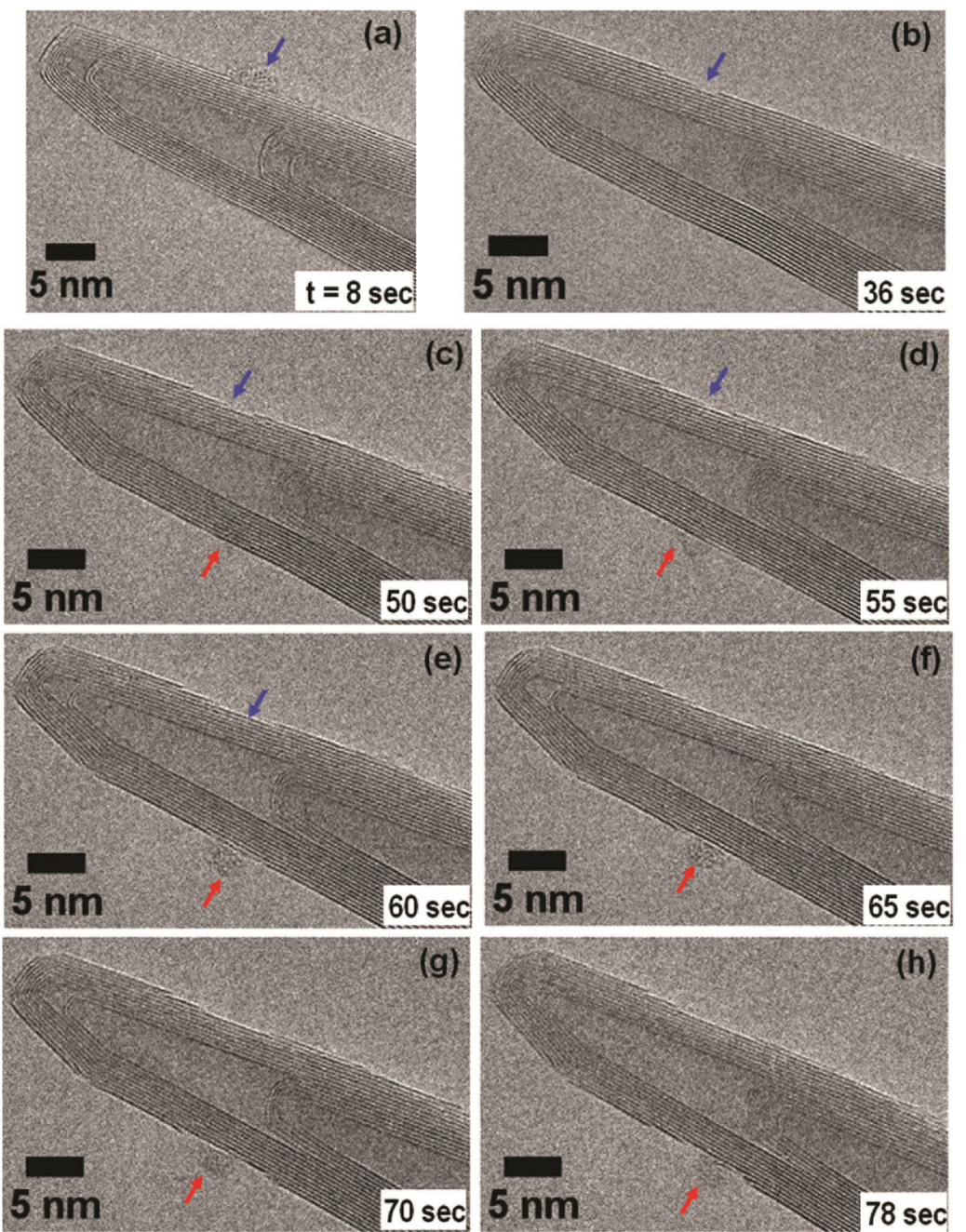

Figure 2.

Side wall damage to a carbon nanotube from electron beam exposure in $0.7 \mathrm{mbar}_{2}$ at room temperature. The blue arrow in (a) and the red arrows in (c) to (h) suggest that the ionized gas first attacks the carbon nanotube side wall by an amorphization process, and leads to the removal of the side walls over time. 

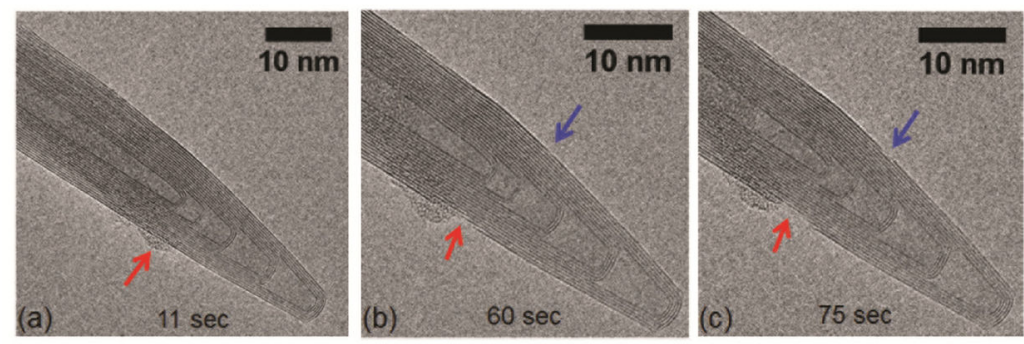

Figure 3.

Attack of side wall of nanotube upon exposure to electron beam in $0.7 \mathrm{mbar}_{2}$ at room temperature. The damage mechanism is similar to that in $\mathrm{O}_{2}$, as illustrated in Figure 2. 

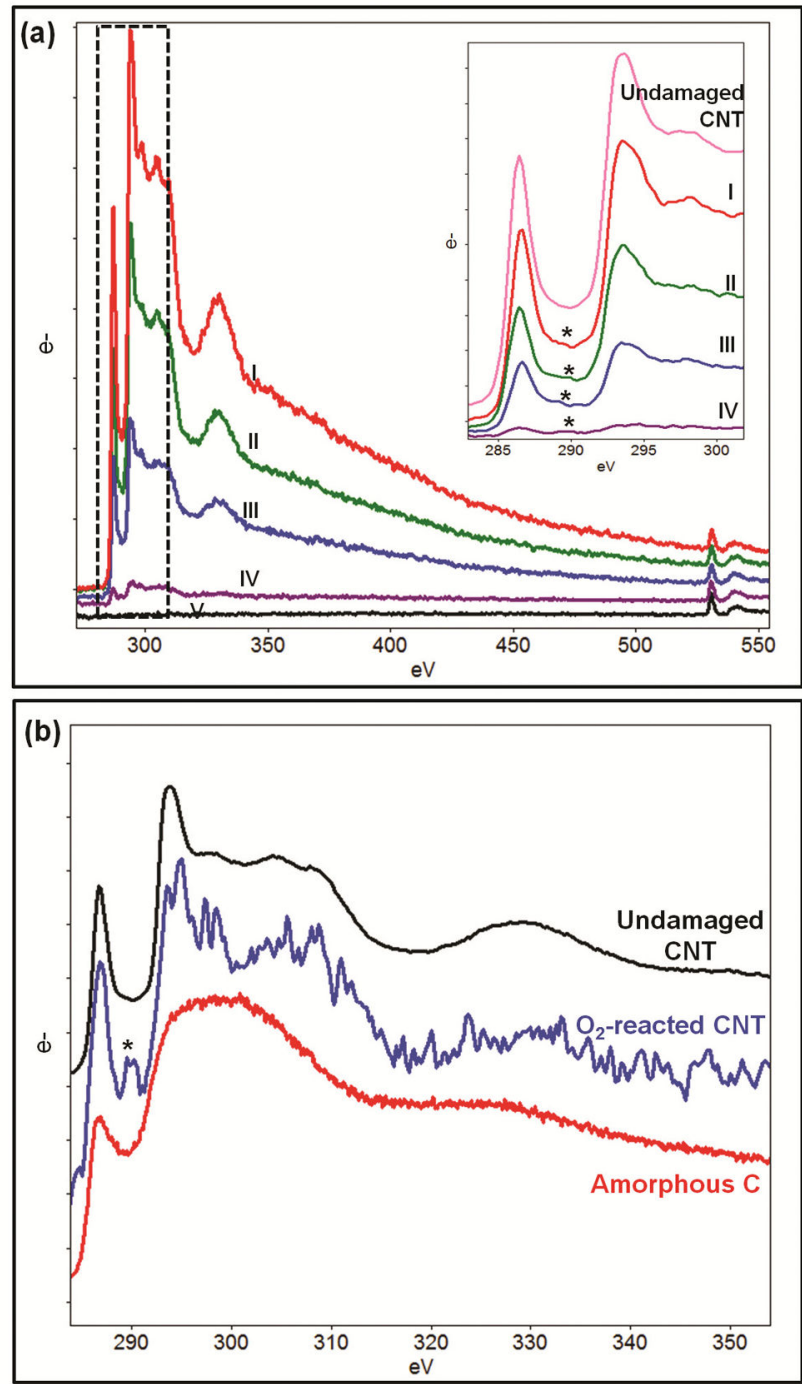

Figure 4.

(a) Time series EEL spectra (I to V) during carbon nanotube ionization in oxygen. The inset of 4(a) shows spectra I to IV and an undamaged nanotube in the energy range of 285 to 300 eV, where additional broad peaks centered around 289-290 eV (marked with *) are observed in spectra I to IV. (b) Comparison of C K EEL spectra from an undamaged nanotube, a CNT which has been reacted in $\mathrm{O}_{2}$ with the electron beam on, and amorphous carbon. 

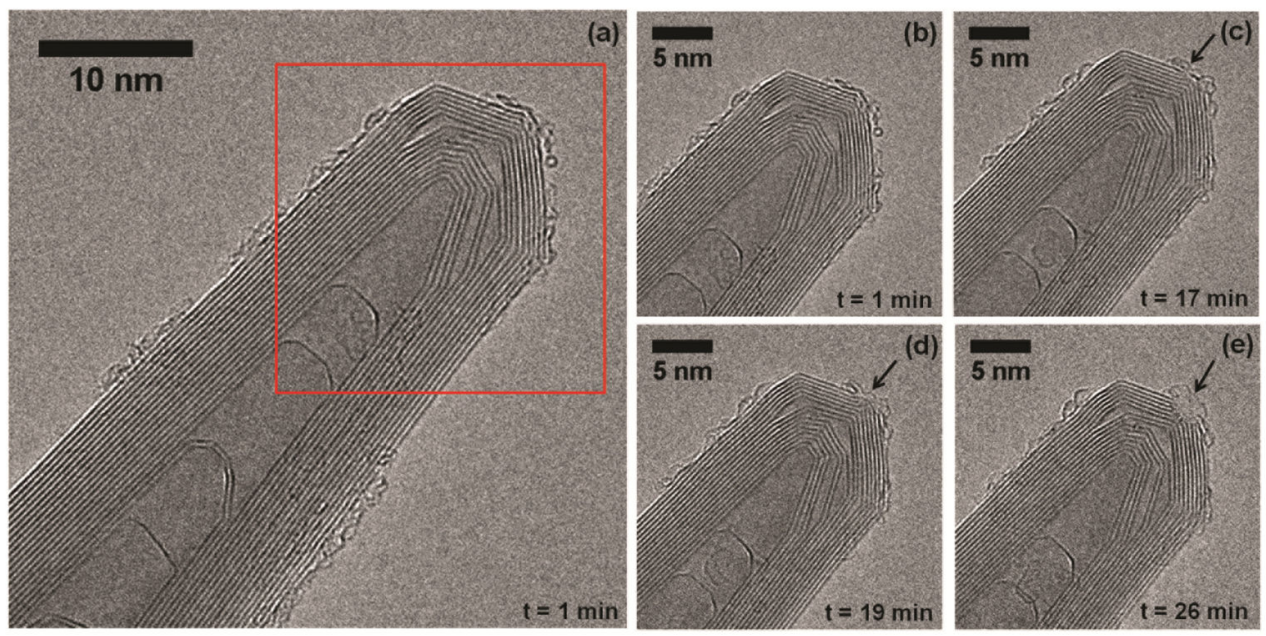

Figure 5.

Structural change in a carbon nanotube during continuous electron irradiation in high vacuum (pressure $\sim 1 \times 10^{-7}$ mbar). (a) Aberration-corrected TEM image of a MWNT. (b) (d) show the inset of the same nanotube upon continuous electron beam irradiation after 1 min, $17 \mathrm{~min}, 19 \mathrm{~min}$ and $26 \mathrm{~min}$. At $17 \mathrm{~min}$, the outermost cap starts opening, and several caps at the tip were destroyed after $26 \mathrm{~min}$. 

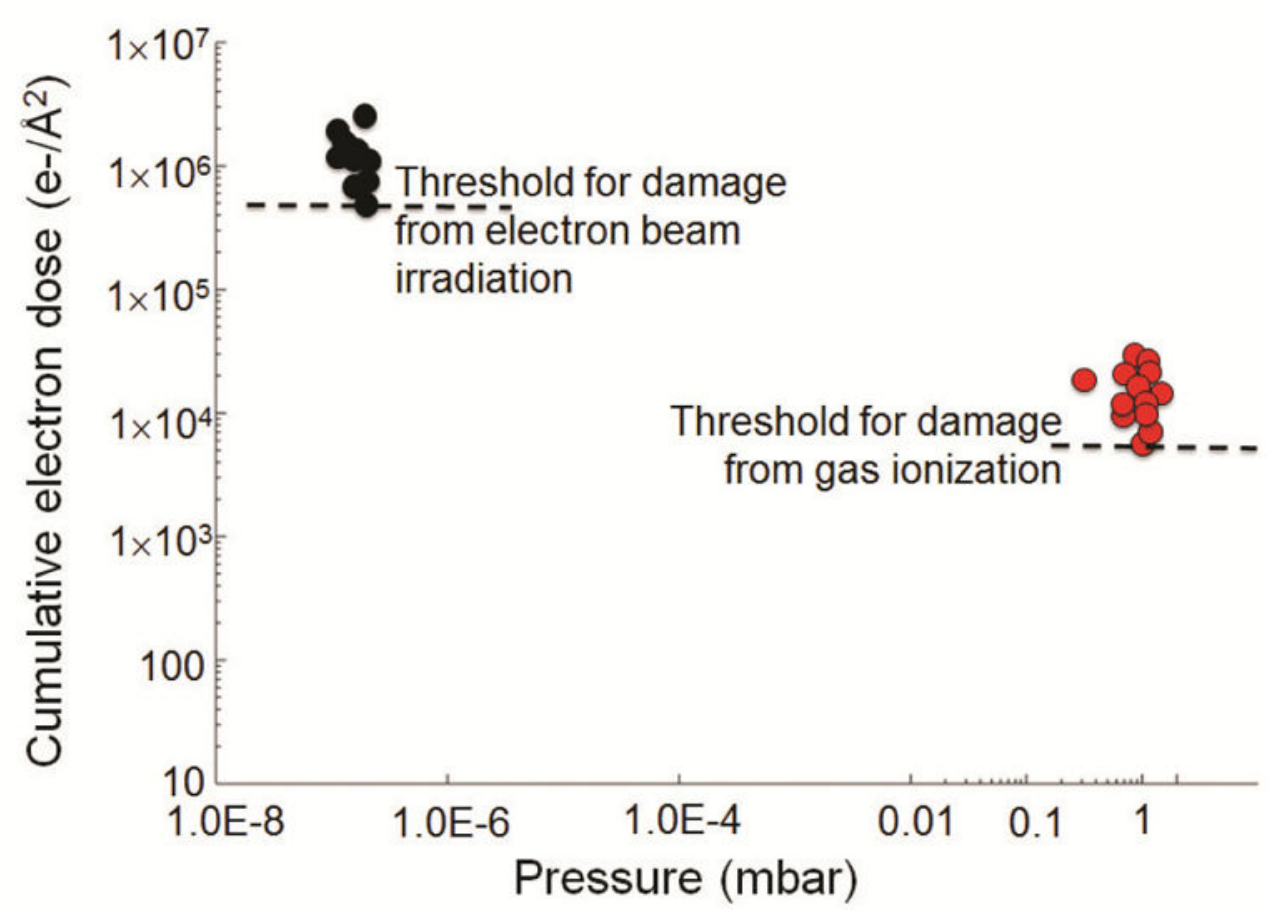

Figure 6.

Cumulative electron dose to damage MWNTs by continuous $80 \mathrm{keV}$ electron beam irradiation in high vacuum and in gas environments in an ETEM at room temperature. 

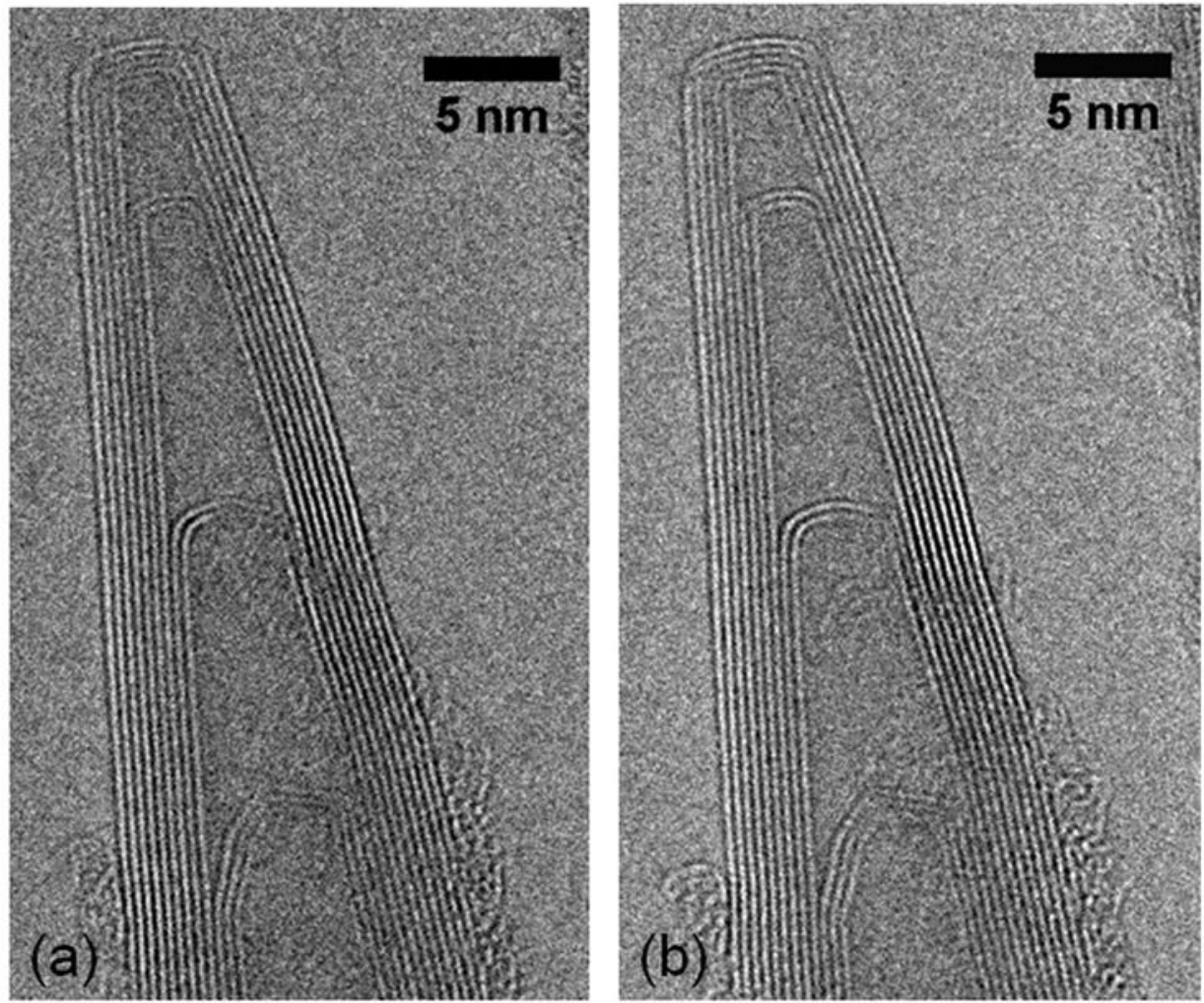

Figure 7.

Aberration-corrected TEM images of the same MWNT (a) at $300{ }^{\circ} \mathrm{C}$ and (b) oxidized at 1.5 mbar $\mathrm{O}_{2}$ at $300{ }^{\circ} \mathrm{C}$ for $15 \mathrm{~min}$ with the beam blanked showing no damage at the cap of the nanotube even after oxidation at elevated temperature. 